\title{
Global surgical experience with the Acorn cardiac support device
}

\author{
Mehmet C. Oz, MD \\ Wolfgang F. Konertz, MD, $\mathrm{PhD}^{\mathrm{b}}$ \\ Franz X. Kleber, MD, PhD ${ }^{\mathrm{C}}$ \\ Friedrich W. Mohr, MD, PhD ${ }^{\mathrm{d}}$ \\ Jan F. Gummert, $M D^{\mathrm{d}}$ \\ Jorg Ostermeyer, $\mathrm{MD}^{\mathrm{e}}$ \\ Michael Lass, MD \\ Jai Raman, MBBS, MMed ${ }^{f}$ \\ Michael A. Acker, MD ${ }^{g}$ \\ Nicholas Smedira, MD ${ }^{\mathrm{h}}$
}

\begin{abstract}
From the Cardiovascular Institute, Columbia-Presbyterian Medical Center, ${ }^{\mathrm{a}}$ New York, NY; Universität Berlin Klinik für Kardiovasculare Chirurgie Charité Universitätsklinikum, ${ }^{\text {b }}$ Berlin, Germany; Klinik für Innere Medizin, Unfallkrankenhaus Berlin, ${ }^{\mathrm{C}}$ Berlin, Germany; Klinik für Herzchirurgie, Herzzentrum Leipzig $\mathrm{GmbH},{ }^{\mathrm{d}}$ Leipzig, Germany; Herzchirurgische Abteilung, Allgemeines Krankenhaus St Georg, ${ }^{\text {e }}$ Hamburg, Germany; Austin Repatriation Medical Centre, ${ }^{\mathrm{f}}$ Heidelberg, Australia; Hospital of the University of Pennsylvania, ${ }^{\mathrm{g}}$ Philadelphia, $\mathrm{Pa}$; and The Cleveland Clinic Foundation, ${ }^{\mathrm{h}}$ Cleveland, Ohio.

Results: At implantation, 11 patients were in New York Heart Association class II, 33 were in class III, and 4 were in class IV. The average CorCap implantation time was 27 minutes. The mean intraoperative reduction in left ventricular end-diastolic dimension was $4.6 \% \pm 1 \%$. There were no device-related intraoperative complications. Eight early and 9 late deaths occurred during follow-up extending to 24 months. Actuarial survival was $73 \%$ at 12 months and $68 \%$ at 24 months. There were no device-related adverse events or evidence of constrictive disease, and coronary artery flow reserve was maintained. Ventricular chamber dimensions decreased, whereas ejection fraction and New York Heart Association class were improved in patients overall and in those patients implanted with the CorCap device without concomitant operations.
\end{abstract}

Received for publication May 13, 2002; revisions requested July 8, 2002; revisions received Oct 21, 2002; accepted for publication Oct 23, 2002.

Address for reprints: Mehmet C. Oz, MD, Columbia-Presbyterian Medical Center, 117 Ft Washington Ave, New York, NY 10032 (E-mail: mco2@columbia.edu).

J Thorac Cardiovasc Surg 2003;126:983-91

Copyright (C) 2003 by The American Association for Thoracic Surgery

$0022-5223 / 2003 \$ 30.00+0$

doi:10.1016/S0022-5223(03)00049-7

Conclusions: The CorCap device appears safe for patients with dilated cardiomyopathy. Randomized clinical trials are underway in Europe, Australia, and North America.

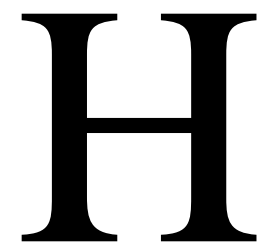

eart failure is characterized by a number of important degenerative changes in cardiac structure and function. Alterations are seen at various levels, including gene expression, cell organelle integrity, and cell shape and size, as well as organization of muscle fibers and extracellular matrix. Changes in structure might be compensatory in the early phases of heart failure ${ }^{1,2}$ but ultimately
Objective: Surgical intervention is an option for treating the remodeled and dilated left ventricles of patients with heart failure. Providing end-diastolic support with an innovative mesh-like cardiac support device reduces mechanical stress, improves function, and reverses cardiac remodeling in animal models without safety issues. The objective of this study was to review the global clinical safety and feasibility experience of this device.

Methods: The Acorn CorCap cardiac support device (Acorn Cardiovascular, Inc, St Paul, Minn) has been implanted worldwide in more than 130 patients with dilated cardiomyopathy with or without concomitant cardiac surgery. The device is positioned around the ventricles and given a custom fit. A series of 48 patients were implanted with the device in initial safety and feasibility studies, of whom 33 also received concomitant cardiac surgery. become maladaptive, contributing to a progressive loss of cardiac function. After an 

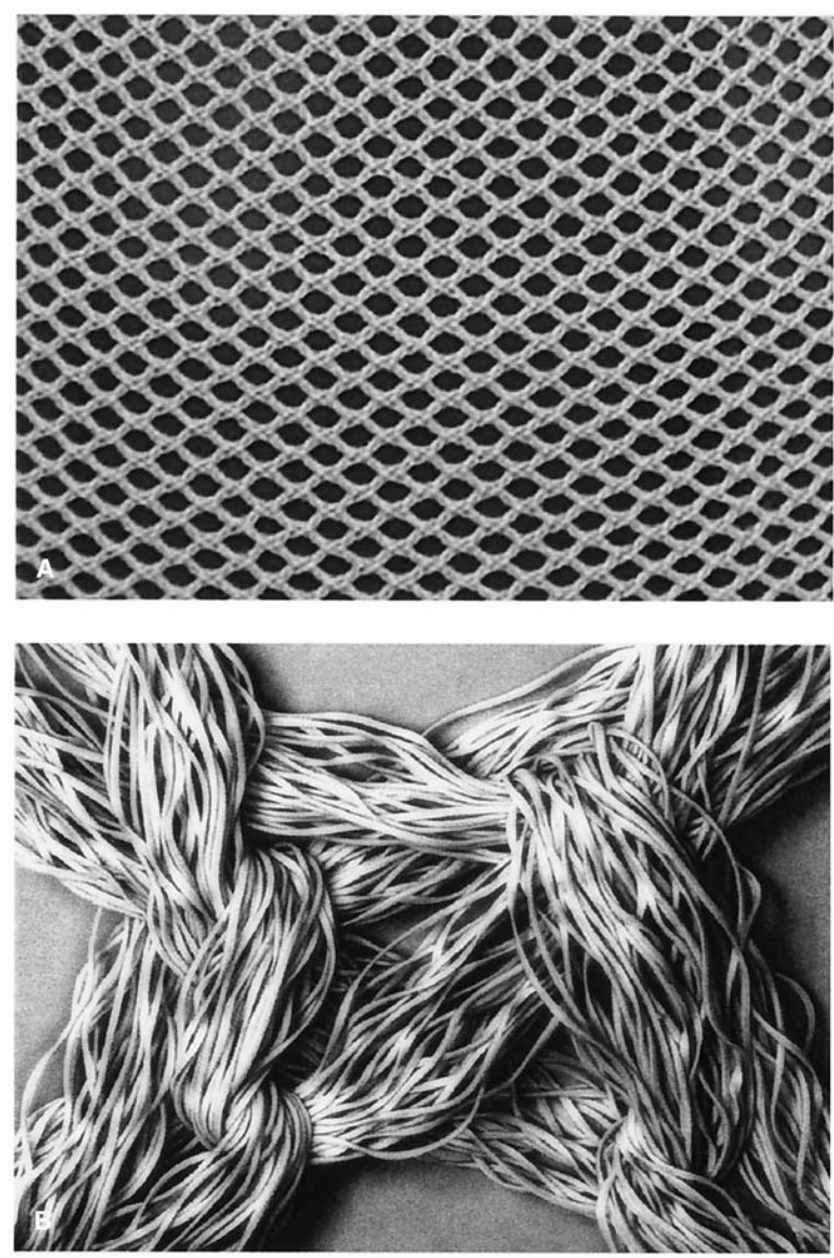

Figure 1. CSD structure. The CSD is a polyester knit construction with compliance characteristics designed to encourage beneficial reverse remodeling in dilated cardiomyopathy: $A$, photograph of an isolated area of open-weave construction (original magnification approximately $2.5 \times$ ); B, scanning electron microscopy photograph showing node (knot; original magnification approximately $70 \times$ ).

initial insult, the heart compensates with a number of acute adaptive mechanisms to maintain adequate cardiac output. Cardiac dilation and activation of neurohormones serve to maintain arterial pressure and perfusion of vital organs. However, dilation increases biomechanical wall stress and cardiac myocyte stretching, which contribute to the development of heart failure. Remodeling of cardiac structure and function in response to injury is a well-established hallmark in the progression of heart failure and is predictive of increased mortality risk. ${ }^{3-6}$

Medical therapy, such as that represented by angiotensinconverting enzyme inhibitors and $\beta$-blockers, is the standard of care for management of heart failure. However, numerous signaling pathways implicated in the promotion and progression of heart failure suggest that drugs might not provide all-encompassing protection against deterioration in cardiac structure and function. Even multidrug regimens might not prevent disease progression, suggesting the need for alternative approaches. Surgical therapies intended to unload the heart have received considerable recent attention. One such means is represented by the Acorn CorCap cardiac support device (CSD; Acorn Cardiovascular, Inc, St Paul, Minn), a mesh-like implantable device that is surgically positioned around the heart and adjusted to provide circumferential diastolic support. The CSD is intended to reduce wall stress and myocyte overstretching during end diastole and periodic hemodynamic overload conditions. By reducing or limiting the stress and stretching on the myocardium, a key component of the remodeling process might be halted or reversed.

\section{Methods}

The CSD is made from a mesh-like polyester fabric (Figure 1). The fabric is constructed from a multifilamentous yarn to provide high-strength and fatigue-resistant characteristics while maintaining flexibility. A knit construction was chosen for the fabric to impart tear and ravel resistance. The device is designed with bidirectional compliance, providing greater circumference support compared with the base-to-apex direction. This has the effect of reshaping the heart to a more ellipsoidal shape. The CSD is available in 6 sizes, with the final fitting performed by the surgeon for the specific heart.

Appropriate patients for CSD implantation are adults with systolic heart failure of ischemic or idiopathic cause with left ventricular dilation. Patients might also require reparative cardiac surgery, such as mitral valve repair-replacement or coronary artery bypass grafting. Their New York Heart Association (NYHA) functional class should be III or early IV. Typically, these patients have debilitating symptoms despite aggressive treatment with cardiac medications or show evidence of deterioration despite their medications.

The upper inclusion limit for ejection fraction in this study was $35 \%$, except for patients receiving concomitant mitral valve surgery, in whom a limit of $45 \%$ was applied. Patients with primary diastolic dysfunction or restrictive disease were excluded. Previous cardiac surgery was not a contraindication, unless patent coronary artery grafts might be compromised by the presence of the CSD or adhesions might prevent proper CSD placement. Patients in endstage NYHA class IV receiving continuous intravenous inotropic agents or mechanical support were excluded.

If the device is used as sole therapy, the CSD can be implanted off pump. The device can also be implanted in conjunction with additional reparative operations on the heart that are done during cardiopulmonary bypass. In these cases device implantation should be started during pump support to facilitate placement of the posterior atrioventricular groove sutures. The final fitting of the device is accomplished with the patient off pump with a full and beating heart to ensure appropriate fitting. Additional techniques involving minimally invasive approaches are in development.

After sternotomy, the pericardium is opened, and the diameter of the left ventricle at the midpapillary muscle level is measured by 


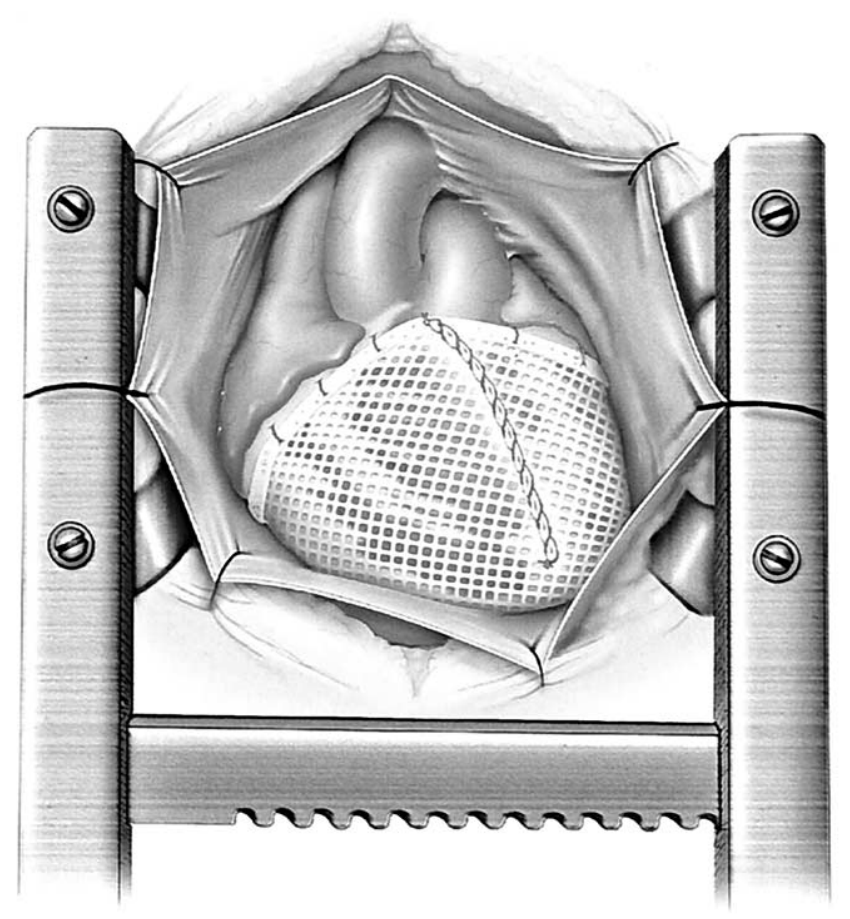

Figure 2. Positioning and securing the CSD. The CSD hemline is attached near the atrioventricular groove through a series of interrupted sutures, starting with the posterior aspect. An anterior seam is used to create the proper device fit.

using transesophageal echocardiography. The appropriate CSD size is selected by measuring the heart's circumference and baseto-apex dimension. A measuring tape or cord is placed around the heart at the base to approximate the circumference, and the length of the heart is measured from the base to the apex anteriorly. These 2 measurements are then used to select the appropriate size device according to a sizing chart provided on the device package.

Once chosen, the CSD is positioned around the ventricles, with particular care to ensure that the hemline lies above or just below the atrioventricular groove. A series of 4-0 polypropylene sutures is used to secure the device in place around the base of the heart, starting in the posterior region and working laterally. Excess fabric is accumulated anteriorly by using a specially designed clamp. The excess material is then removed, and a new anterior seam is formed (Figure 2). We seek to obtain a snug fit, which might result in a slight reduction in the diameter of the heart, as measured by means of transesophageal echocardiography, but avoids any reduction greater than $10 \%$ to minimize the potential for any adverse events related to device fit. This snug fit ensures that the CSD relieves a portion of mechanical stress and stretching in the ventricular wall.

As of April 1, 2002, a total of 130 patients have received the CSD. Patients include various safety study participants and those enrolled in global randomized clinical trials currently underway in Europe and the United States. Results from the randomized trials will not be available until study enrollment and follow-up are complete. Thus this report will focus on patients receiving the CSD

\section{TABLE 1. Major inclusion and exclusion criteria}

\begin{tabular}{lc}
\hline Inclusion & Exclusion \\
\hline Dilated cardiomyopathy: & Current or anticipated need \\
ischemic or nonischemic & for LVAD or total \\
Stable and optimal medical & artificial heart \\
management & Likelihood of existing \\
Adult (18-80 y) & cardiothoracic adhesions \\
Indexed LVEDD $\geq 30 \mathrm{~mm} / \mathrm{mm}^{2}$ & preventing proper device \\
LVEF $\leq 35 \%$ or LVEF $\leq 45 \%$ for & placement \\
CSD valve surgery & Patent CABG \\
History of NYHA III or early & NYHA IV dependent on \\
stage IV & intravenous inotropes \\
Acceptable renal function & NYHA IV dependent on \\
(creatinine $<3.5 \mathrm{ml} / \mathrm{dL}$ ), & intravenous inotropes \\
hepatic function (SGOT and & Late-stage heart failure \\
SGPT $<2 \times$ normal), & with increased surgical \\
pulmonary function (FEV 1 & risk \\
$>1.5 \mathrm{~L}$ ) & Acute myocardial infarction \\
& within 3 mo \\
& Hemodynamically unstable \\
& or uncontrolled \\
& arrhythmias \\
& Comorbid condition with \\
& life expectancy $<2$ y
\end{tabular}

SGOT, Aspartate aminotransferase; SGPT, alanine aminotransferase; $F E V_{1}$, forced expiratory volume in 1 second; $L V A D$, left ventricular assist device; $C A B G$, coronary artery bypass grafting.

who are not enrolled in these randomized trials. Ethics committee approval was obtained at participating institutions before entry of patients into this safety study.

Initial safety studies included a series of 29 patients enrolled at Charité Universitätsklinikum, Humboldt-Universität zu, Berlin, Germany, between April 1999 and July 2000. Of the 29 patients, 12 required no additional cardiac surgery other than the CSD implantation, and the remainder had some type of concomitant cardiac surgery. The majority of these procedures were mitral valve repairs or replacements. The initial safety studies also included a group of 5 patients with ischemic heart disease who were enrolled at the Austin and Repatriation Medical Centre, Melbourne, Australia. Coronary artery bypass grafting was performed on all of the patients concomitant with CSD implantation. None of these patients required valve repair or replacement. Coronary artery angiography was performed at 6 months' follow-up to evaluate graft patency.

A final group of 14 nonrandomized patients was also included. Three patients required no concomitant surgery; 7 underwent valve repair or replacement, 2 had coronary artery bypass operations, and 2 had both bypass grafting and valve repair or replacement. All of these groups combine for a total of 48 initial safety study patients. Results are reported as means \pm SD (range) unless otherwise stated.

Inclusion and exclusion criteria for patients in these safety studies are summarized in Table 1. During the initial experience, it became important to exclude patients with very advanced or latestage heart failure because of a very high operative risk. A series 


\section{TABLE 2. Late-stage heart failure relative risk factors}

Late-stage heart failure with increased surgical risk as defined by the presence of $\geq 4$ of the following relative risk factors:

- LVEDD $\geq 80 \mathrm{~mm}$

- Peak $\mathrm{VO}_{2} \leq 13 \mathrm{~mL} \cdot \mathrm{kg}^{-1} \cdot \min ^{-1}$ (cardiopulmonary exercise test)

- Resting systolic BP $\leq 80 \mathrm{~mm} \mathrm{Hg}$ (on clinical examination)

- Atrial fibrillation at time of enrollment or paced rhythm with underlying atrial fibrillation

- Heart failure duration $\geq 8$ y

- Exercise-induced increase in systolic BP $\leq 10 \%$ (cardiopulmonary exercise test)

- 6-min walk $\leq 350$ m (1148 feet)

- Previous cardiac surgery

- BUN $\geq 100 \mathrm{mg} / \mathrm{dL}$

- Cachexia (clinical impression)

Peak $\mathrm{VO}_{2}$, Peak oxygen uptake; $B P$, blood pressure; $B U N$, blood urea nitrogen.

of 10 criteria were determined, representing late-stage heart failure (Table 2). Patients with 4 or more of these criteria were excluded from the study.

Patient follow-up was scheduled for 3, 6, 12, and 18 to 24 months after CSD implantation. Evaluation and testing included clinical assessment, echocardiography (left ventricular end-diastolic dimension [LVEDD], left ventricular end-systolic dimension, and left ventricular ejection fraction $[\mathrm{LVEF}])$, and recording of any adverse events. Quality-of-life instruments included the Minnesota Living with Heart Failure Questionnaire ${ }^{7,8}$ and the Uniscale (visual analog scale). ${ }^{9}$ A group of 15 patients was also entered into a substudy to investigate the effect of the CSD on diastolic function. Baseline cardiac catheterization was performed before device implantation and at 3, 6, and 12 months' follow-up. Simultaneous right and left ventricular pressures were analyzed, and pressure-volume curves were constructed by using micromanometer tip left ventricular pressure recordings and biplane contrast ventriculography. ${ }^{10,11}$ In addition, coronary flow was measured by using a 0.014-inch Doppler flow wire placed in the proximal left coronary artery before and after infusion of $18 \mu \mathrm{g}$ of adenosine. Coronary flow reserve was calculated as the ratio of average peak velocity during maximum hyperemia divided by baseline average peak velocity. ${ }^{12,13}$

\section{Results}

Patients implanted with the CSD in the nonrandomized studies were primarily male ( $88 \%)$, with an age at the time of operation of $59.3 \pm 10.1$ years (range, 34-87 years). Idiopathic dilated cardiomyopathy predominated $(\mathrm{n}=35$ [73\%]), and the remainder of the patients had an ischemic cause $(\mathrm{n}=13[27 \%])$. NYHA functional class at enrollment was $2.9 \pm 0.5$ (range, 2-4), although all patients had a history of at least one episode of functional class III or IV. A significant degree of cardiac remodeling and loss of cardiac function was apparent in these patients at baseline, with an LVEDD of $71.3 \pm 7.3 \mathrm{~mm}$ (range, $59-86 \mathrm{~mm}$ ) and an LVEF of $22.3 \% \pm 8.9 \%$ (range, $10 \%-41 \%$ ). Patients had
TABLE 3. Operative mortality and late deaths

\begin{tabular}{lccc}
\hline Patient group (n) & Operative mortality & Late deaths & Total \\
\hline CSD only (15) & 1 & 4 & 5 \\
Concomitant (33) & 7 & 5 & 12 \\
All patients (48) & 8 & 9 & 17 \\
\hline
\end{tabular}

experienced an average of $2.0 \pm 1.8$ cardiac hospitalizations during the year preceding the operation and were receiving a stable course of medication for at least a month before CSD implantation.

The complete surgical procedure required an average of $2.3 \pm 0.6$ hours (range, 1.6-3.7 hours) for CSD-only operations compared with $3.5 \pm 1.2$ hours (range, 2.0-6.7 hours) for procedures involving concomitant cardiac operations. The average implantation time was $23.1 \pm 6.1$ minutes (range, 15-40 minutes) for patients receiving only the CSD, whereas implantation time in conjunction with concomitant cardiac procedures was slightly greater $(29 \pm 11$ minutes [range, 14-67 minutes]). Crossclamp time was not required for CSD-only implantation, and $49.7 \pm 30.3$ minutes (range, 21-125 minutes) of crossclamp time was used in conjunction with concomitant procedures. Total cardiopulmonary bypass time was $24.7 \pm 9$ minutes (range, 20-32 minutes) for CSD-only procedures and $94.8 \pm 36$ minutes (range, 55-188 minutes) for concomitant procedures. Mean intraoperative LVEDD reduction was $3.2 \mathrm{~mm}$ (4.6\%). There were no device-related intraoperative complications in this study.

There were 8 deaths attributed to operative mortality (Table 3), having taken place during the first 30 days after the operation or during the initial hospitalization. This included $1(7 \%)$ of 15 patients in the CSD-only group and 7 (21\%) of 33 patients in the concomitant surgery group. There were 9 late deaths in total, including several not related to heart failure (cancer in 2 patients and suboptimal anticoagulant level in a patient with a prosthetic mechanical heart valve). Other late deaths were attributed to pneumonia, ventricular arrhythmia, progressive recurrent mitral regurgitation, and cerebral hemorrhage. The 2 remaining long-term deaths were considered cardiac failure, with 1 occurring approximately 1 month after weaning from a left ventricular assist device. Of the 17 total deaths in the series, $5(38 \%)$ occurred in patients with an ischemic cause, and 12 (34\%) occurred in patients with an idiopathic cause. No deaths or adverse events were CSD related.

Because of deaths and other factors, follow-up was not obtainable for patients at all time points. At 12 months, 12 patients had died. In addition, one patient was terminated from the study before 12 months' follow-up as a consequence of a stroke. Four patients missed the 12-month examination, 4 patients were permanently lost to follow-up, and 2 patients had not yet reached the 12-month time point. 


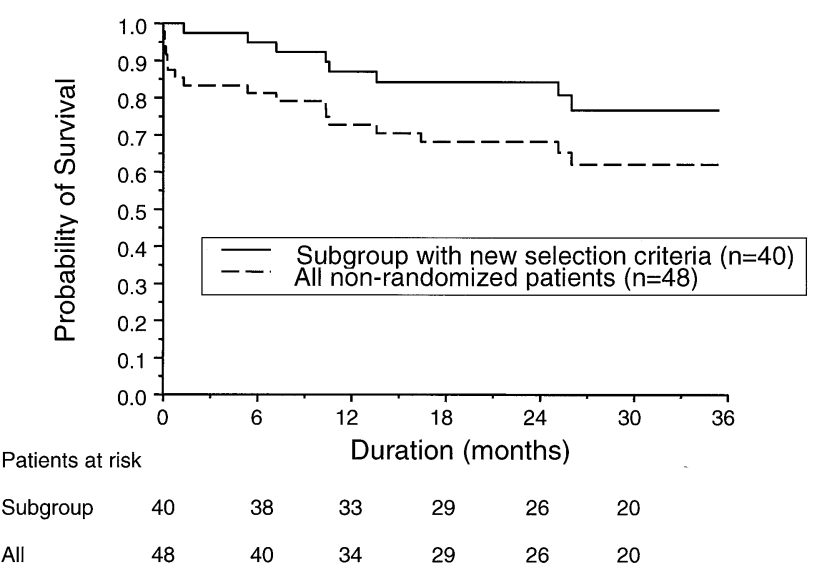

Figure 3. Kaplan-Meier survival curves for all patients in the series $(n=48)$ and a subgroup $(n=40)$ in which patients at high surgical risk are screened out according to revised selection criteria.

Thus, at 12 months, data were available for $25(52 \%)$ of 48 patients.

Various factors were likely active in influencing patient outcome. Among these were patient selection criteria. In light of the operative mortality experienced in this series, alterations were made in selection criteria (see late-stage heart failure criteria) to screen out patients at unacceptably high surgical risk. With the revised selection criteria, patients representing 7 of the 8 early deaths and 1 of the 9 late deaths would have been screened out of the study. KaplanMeier survival curves in Figure 3 demonstrate that analysis, in which the lower curve represents all 48 patients, and the upper curve excludes deaths of patients who would have been screened out by the revised selection criteria. In addition, implantation techniques were refined, including fitting the device with no greater than a $10 \%$ reduction in LVEDD. The modified criteria are also the basis for enrollment of patients in randomized trials currently underway with this device.

At this time, average implantation duration is nearly 24 months for patients in the study, with a mean follow-up of 20 months for all nonrandomized patients and a minimum follow-up of 10 months on current survivors. Follow-up evaluations on these nonrandomized patients up to 24 months are encouraging and demonstrate that the CSD effectively limits progressive cardiac dilation and decreased cardiac function. Average heart size was shown to decrease by statistically significant amounts at 12 months' follow-up, whereas average LVEF increased (Tables 4 and 5). ${ }^{14}$ Functional status and quality-of-life assessments, including NYHA functional class, the Minnesota Living with Heart Failure Questionnaire, and the Uniscale, all point toward improvement in quality of life for these patients. Notably, the decreases in heart size and improvements in ejection
TABLE 4. All patients at 12 mo follow-up

\begin{tabular}{lcc}
\hline & $\begin{array}{c}\text { Before } \\
\text { implantation }\end{array}$ & 12 $\mathbf{~ m o}$ \\
\hline LVEDD, mm $(\mathrm{n}=25)^{*}$ & $72.8 \pm 7.1$ & $64.1 \pm 10.6 \dagger$ \\
LVESD, mm $(\mathrm{n}=23)^{*}$ & $62.8 \pm 8.1$ & $54.7 \pm 12.0 \dagger$ \\
LVEF, \% $(\mathrm{n}=23)^{*}$ & $23.6 \pm 8.0$ & $29.9 \pm 12.2 \dagger$ \\
MR, 0-4 $+(\mathrm{n}=22) \ddagger$ & $1.6 \pm 1.0$ & $0.5 \pm 0.7 \dagger$ \\
NYHA $(\mathrm{n}=26) \ddagger$ & $2.7 \pm 0.5$ & $1.7 \pm 0.7 \dagger$ \\
MN Living with HF $(\mathrm{n}=20) \ddagger$ & $30.9 \pm 19.6$ & $25.0 \pm 21.1$ \\
Uniscale $(\mathrm{n}=18) \ddagger$ & $4.6 \pm 2.3$ & $6.3 \pm 2.2 \dagger$
\end{tabular}

Mean \pm SD. LVESD, Left ventricular end-systolic dimension; $M R$, mitral regurgitation; $M N$ Living with $H F$, Minnesota Living with Heart Failure Questionnaire; Uniscale, Quality of Life Questionnaire.

*Paired $t$ test.

$\dagger P \leq .05$

‡Wilcoxon signed-rank test.

TABLE 5. CSD-only patients at 12 mo follow-up

\begin{tabular}{|c|c|c|}
\hline & $\begin{array}{c}\text { Before } \\
\text { implantation }\end{array}$ & $12 \mathrm{mo}$ \\
\hline LVEDD, $\mathrm{mm},(\mathrm{n}=12)^{*}$ & $72.7 \pm 5.5$ & $64.4 \pm 5.4 \dagger$ \\
\hline LVESD, $\mathrm{mm}(\mathrm{n}=12)^{*}$ & $64.4 \pm 5.4$ & $56.9 \pm 5.7 \dagger$ \\
\hline LVEF, $\%(n=12)^{*}$ & $22.7 \pm 5.7$ & $28.6 \pm 8.2 \dagger$ \\
\hline MR, $0-4+(n=12)^{*}$ & $1.1 \pm 0.5$ & $0.5 \pm 0.8$ \\
\hline NYHA (n = 12)‡ & $2.5 \pm 0.5$ & $1.8 \pm 0.7 \dagger$ \\
\hline MN Living with HF ( $\mathrm{n}=10) \ddagger$ & $34.3 \pm 21.6$ & $28.3 \pm 18.6$ \\
\hline Uniscale $(\mathrm{n}=10) \ddagger$ & $4.5 \pm 2.2$ & $6.1 \pm 2.2 \dagger$ \\
\hline
\end{tabular}

Mean $\pm \mathrm{SD}$. LVESD, Left ventricular end-systolic dimension; MR, mitral regurgitation; $M N$ Living with $H F$, Minnesota Living with Heart Failure Questionnaire; Uniscale, quality-of-life questionnaire.

*Paired $t$ test.

$\dagger P \leq .05$.

$\ddagger$ Wilcoxon signed-rank test.

fraction appear to be maintained at 18 to 24 months after the operation (Figure 4). Encouraging results were seen for the overall patient cohort, as well as for the subgroup of patients who received only the CSD (Table 5).

Follow-up results obtained from the safety study patients in Melbourne with ischemic heart disease and requiring coronary artery bypass surgery are consistent with the results obtained for patients receiving the CSD in a standalone procedure and those patients who required concomitant mitral valve repair or replacement. In these ischemic patients, the CSD prevented cardiac dilation and was well tolerated. ${ }^{15,16}$ All coronary artery bypass grafts were patent at follow-up, and cardiac symptoms were reduced in all patients. No device-related adverse events have occurred in this group of patients.

A subset of study patients underwent hemodynamic and pressure-volume loop analyses at 3, 6, and 12 months' follow-up. Values obtained for the differences in ventricular end-diastolic pressure, right atrial mean pressure, and right ventricular pressure ratio revealed no indication of cardiac 


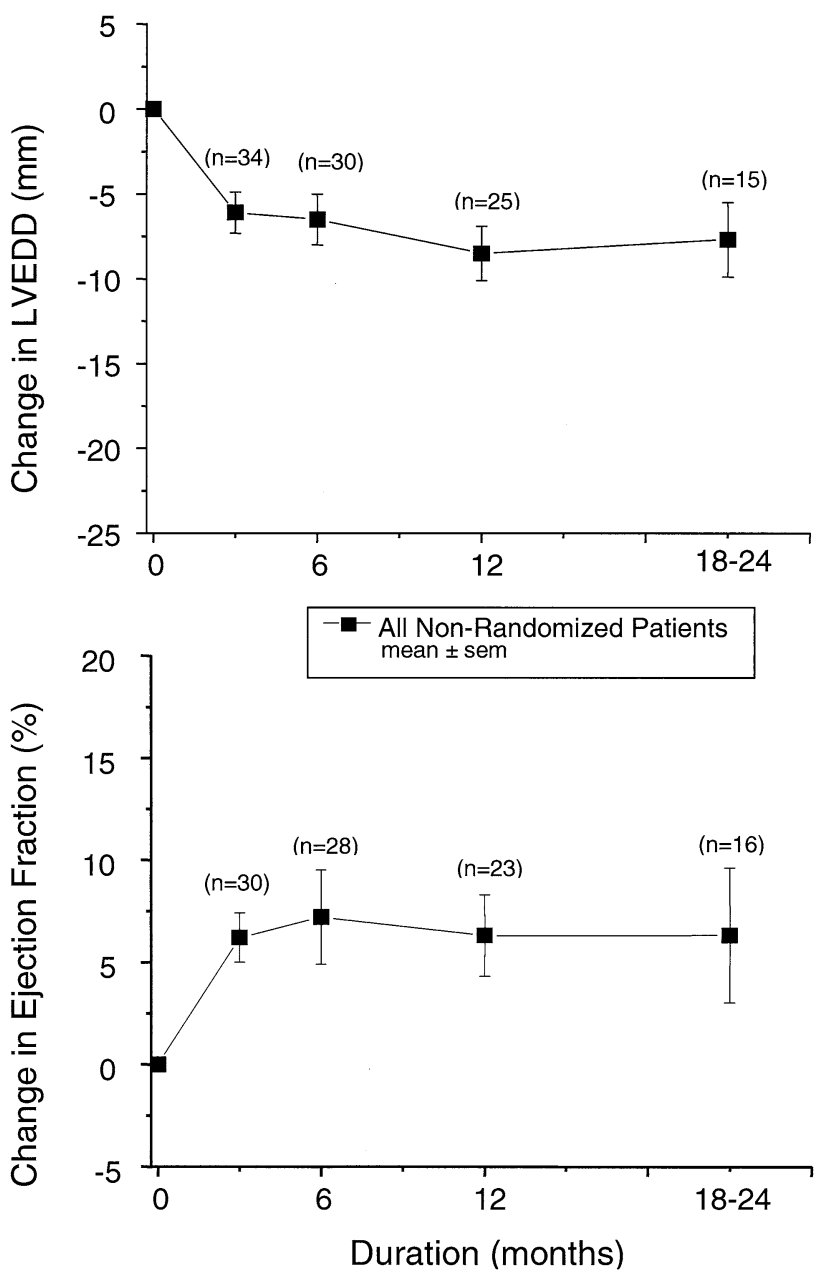

Figure 4. Changes in LVEDD and LVEF at follow-up. The mean \pm SEM changes in LVEDD (in millimeters) and LVEF (percentage) during follow-up are shown, along with the numbers of patients at each time point for whom baseline and follow-up values were available. The final follow-up (18-24 months) represents combined data for patients with latest follow-up at 18 months $(n=4)$ or 2 years $(\mathbf{n}=\mathbf{9})$.

constriction or diastolic dysfunction (Table 6). Left ventricular pressure-volume analysis supported these findings. Figure 5 depicts pressure-volume loop data for a patient implanted with the device only, with no other concomitant cardiac procedures. These results are representative of the series as a whole ${ }^{17}$ and illustrate a lack of diastolic impairment or development of constrictive physiology.

Coronary flow capacity was evaluated by using intracoronary adenosine administration. All patients studied demonstrated a normal coronary flow reserve with no flow impairment (Table 7). Most patients were receiving an extensive list of cardiac medications, including angiotensinconverting enzyme inhibitor/angiotensin receptor blocker

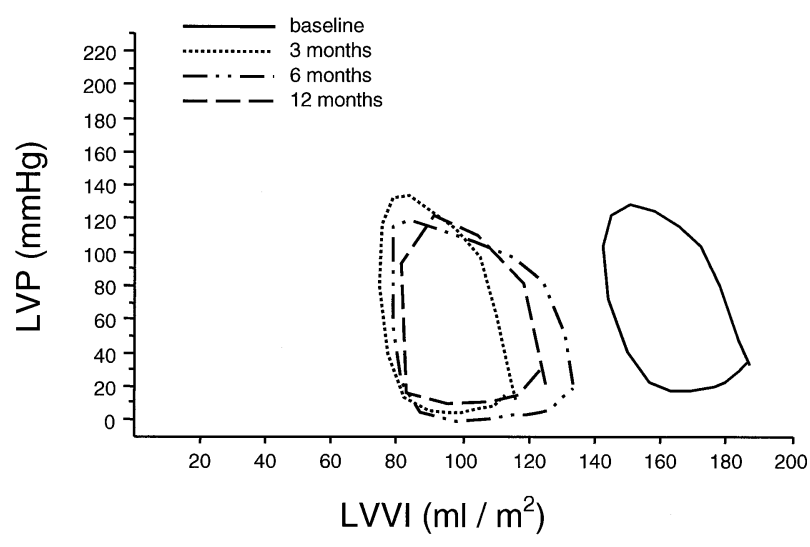

Figure 5. Pressure-volume loop analysis. Cardiac catheterization and biplane contrast ventriculography were used to capture simultaneous pressure-volume data at baseline and follow-up through 12 months. Representative results for a device-only patient are plotted as pressure-volume loops, demonstrating decreases in end-systolic and end-diastolic volumes and a trend toward lower end-diastolic pressure at follow-up.

TABLE 6. Hemodynamic assessment of constriction; 3-, 6-, and 12-month follow-up

\begin{tabular}{lccc}
\hline & \multicolumn{3}{c}{ Follow-up } \\
\cline { 2 - 4 } & $\begin{array}{c}\mathbf{3} \mathbf{~ m o} \\
(\mathbf{n}=\mathbf{1 5})\end{array}$ & $\begin{array}{c}\mathbf{6} \mathbf{~ m o} \\
(\mathbf{n}=\mathbf{1 1})\end{array}$ & $\begin{array}{c}\mathbf{1 2} \mathbf{~ m o} \\
\mathbf{( n = 6 )}\end{array}$ \\
\hline LVEDP-RVEDP (mm Hg) & $5.9 \pm 5.3$ & $5.3 \pm 4.5$ & $7.6 \pm 7.1$ \\
RV ESEDP ratio & $0.28 \pm 0.11$ & $0.38 \pm 0.08$ & $0.32 \pm 0.21$ \\
RAMP (mm Hg) & $8.7 \pm 5.6$ & $11.0 \pm 4.6$ & $10.2 \pm 6.9$ \\
\hline
\end{tabular}

$\overline{M e a n} \pm \mathrm{SD}$. LVEDP, Left ventricular end-diastolic pressure; RVEDP, right ventricular end-diastolic pressure; RV ESEDP, ratio of right ventricular end-systolic pressure/right ventricular end-diastolic pressure; $R A M P$, right atrial mean pressure.

and $\beta$-blockers (Table 8). During follow-up, these medications were generally maintained.

\section{Discussion}

Progressive heart failure is characterized by loss of cardiac function associated with maladaptive changes in myocardial gene expression and neuroendocrine activity, leading to progressive increases in heart size. Increased ventricular wall stress and myocardial stretching resulting from this increase in chamber size are thought to play a role in furthering development toward end-stage disease. Reduction of wall stress and myocardial stretching might be important means for mitigating heart failure progression. One possible approach to accomplish this goal is through passive containment of the heart with the CSD. Results from preclinical and clinical evaluation support this premise.

Chaudhry and colleagues ${ }^{18}$ have reported reduced left ventricular volumes and improved cardiac function param- 
TABLE 7. Coronary flow reserve

\begin{tabular}{lccc}
\hline & \multicolumn{3}{c}{ Follow-up (mo) } \\
\cline { 2 - 4 } & $\mathbf{3}(\mathbf{n}=\mathbf{1 1})$ & $\mathbf{6}$ (n=7) & $\mathbf{1 2}(\mathbf{n}=\mathbf{4})$ \\
\hline Baseline APV $(\mathrm{cm} / \mathrm{s})$ & $25.0 \pm 8.0$ & $24.0 \pm 12.0$ & $28.0 \pm 8.0$ \\
APV after adenosine $(\mathrm{cm} / \mathrm{s})$ & $71.0 \pm 19.0$ & $64.0 \pm 18.0$ & $88.0 \pm 18.0$ \\
Coronary flow reserve* & $2.9 \pm 0.6$ & $3.1 \pm 0.9$ & $3.2 \pm 0.4$ \\
& (range, 2.1-3.7) & (range, 2.2-4.4) & (range, 2.8-3.7) \\
\hline
\end{tabular}

Mean \pm SD

$A P V$, Average peak velocity.

${ }^{*}$ Normal values are greater than 2.0 .

eters in CSD-treated animals by using a microembolic canine heart failure model. At 3 months' follow-up, left ventricular end-diastolic volume (LVEDV) was reduced ( $68 \pm$ $4 \mathrm{~mL}$ at baseline vs $61 \pm 4 \mathrm{~mL}$ at 3 months [mean \pm SEM], $P<.005)$ but increased in the untreated control group (67 $\pm 5 \mathrm{~mL}$ at baseline vs $83 \pm 8 \mathrm{~mL}$ at 3 months). LVEF was increased $(34 \% \pm 1 \%$ at baseline vs $41 \% \pm 1 \%$ at 3 months, $P<.005)$ in animals with heart failure treated with the CSD but decreased in the control group $(36 \% \pm 1 \%$ at baseline vs $28 \% \pm 2 \%$ at 3 months). Similar results have been demonstrated at 6 months' follow-up.

LVEDV and LVEF at follow-up were statistically different between the control and CSD treatment groups. Also, at 3 months' follow-up, regional wall motion was greater in the CSD-treated group compared with that in the control heart failure group, whereas mitral valve regurgitation was eliminated in the treatment group. A trend toward increased left ventricular end-diastolic pressure in the control group and a decrease in the treatment group at follow-up was also noted.

Power and associates ${ }^{19}$ used a high-rate pacing ovine model of heart failure and reported similar findings. Heart size (left ventricular diastolic area) was maintained or reduced with CSD treatment in moderate and more advanced heart failure, despite continued high-rate pacing. Cardiac function also improved with CSD treatment, as reflected by improved LVEF, fractional shortening, positive $\mathrm{dP} / \mathrm{dt}$, and negative $\mathrm{dP} / \mathrm{dt}$. Functional improvement was noted irrespective of whether the CSD was implanted in patients with moderate or more advanced heart failure.

Histomorphologic analysis was performed on cardiac tissue samples from dogs with microemboli-induced heart failure at 3 months' follow-up. Compared with the control group, the tissue from the CSD-treated study group showed less myocyte hypertrophy, less interstitial fibrosis, and greater capillary density. ${ }^{20}$ Further testing in this animal model demonstrated enhanced myocyte function (percentage of shortening and peak derivative of shortening and relaxation) in the CSD-treated group compared with that in the control group. ${ }^{20}$

Biochemical factors implicated in possible mechanisms of actions for CSD therapy have been examined. Sabbah
TABLE 8. Cardiac medications at baseline and 12 months

\begin{tabular}{lcc}
\hline Cardiac medications & $\begin{array}{c}\text { Baseline } \\
\text { (\% of patients) }\end{array}$ & $\begin{array}{c}\mathbf{1 2} \text { mo } \\
\text { (\% of patients) }\end{array}$ \\
\hline ACEI/ARB & $90 / 8$ & $82 / 14$ \\
Diuretic & 98 & 95 \\
Digoxin & 72 & 73 \\
$\beta$-Blocker & 68 & 82 \\
Amiodarone & 19 & 9 \\
Other & 72 & 59
\end{tabular}

$A C E I$, Angiotensin-converting enzyme inhibitor; $A R B$, angiotensin receptor blocker.

and coworkers ${ }^{21}$ reported that stretch protein levels (p21ras, $\mathrm{c}$-fos, and $\mathrm{p} 38 \alpha / \beta \mathrm{MAP}$ kinase) were at lower levels in the CSD-treated group, and $\mathrm{Ca}^{2+}$ cycling was improved (on the basis of an increase in the ratio of $\mathrm{Ca}^{2+}$ ATPase/phospholamban and a downregulation of protein phosphatase activity) in the CSD-treated group. ${ }^{22}$ In addition, the expression ratio of $\mathrm{Bcl}-2 / \mathrm{Bax}$ was altered after CSD implantation, suggesting an attenuation in proapoptotic signaling in cardiomyocytes. ${ }^{23}$ These findings suggest that the CSD can prevent or minimize maladaptive gene expression and phenotypic transformation. In summary, these studies indicate that the CSD, by supporting the ventricle and reducing stress-mediated myocardial stretching, can halt progressive remodeling and perhaps allow for reverse remodeling.

Preclinical studies indicate no signs of a constrictive pattern on the basis of analysis of filling pressures and pressure-volume loops. ${ }^{24}$ In addition, animals with heart failure evaluated at 3 months after CSD implantation demonstrated a normal response to severe volume load by increasing LVEDV, LVEF, and left ventricular stroke volume. $^{25}$

Clinical study results also indicate that patients receiving the CSD do not exhibit a constrictive physiology. As part of the safety study, 15 patients were entered into a substudy designed to address this question. ${ }^{26}$ Patients underwent pressure-volume loop analysis and detailed hemodynamic monitoring, including assessment of left and right ventricular end-diastolic pressures, right atrial pressures, right ventricular pressure ratios, and right ventricular indices. $\mathrm{Pa}$ - 
tients were followed up to 12 months after CSD implantation. On the basis of these analyses, there was no evidence of constriction. In addition, coronary flow reserve measurements conducted in these patients showed normal coronary flow without signs of coronary impairment.

Tissue reaction between the CSD and the heart does not appear to contribute to a constrictive physiology and should not interfere with most future cardiac surgeries. Nevertheless, surface fibrosis associated with the CSD might complicate future cardiac surgeries and might interfere with visualization of the cardiac architecture, including epicardial coronary arteries should a redo operation, such as coronary artery bypass, be required. Experience in animal models suggests that the normal fibrotic response to the CSD is self-limiting and thin in cross-section, and limited clinical experience suggests a similar response in human subjects. However, adequate clinical data are not yet available to determine the ease of access to coronary arteries or other cardiac structures if a future redo operation is required.

Coronary artery bypass graft surgery can be safely performed in conjunction with CSD implantation. After positioning the CSD, small openings are created in the device fabric to access anastomotic sites on epicardial coronary arteries. In a limited series of patients undergoing coronary artery bypass grafting, graft patency was evaluated by means of coronary angiography 6 months after the operation, at which time all grafts remained patent. ${ }^{8}$

Patients with end-stage cardiomyopathy frequently experience severe mitral regurgitation, which contributes to heart failure progression and is predictive of poor outcome. ${ }^{27}$ Mitral regurgitation in the context of cardiomyopathy is believed to result from dilation of the mitral annularventricular apparatus with altered ventricular geometry or from ischemic papillary muscle dysfunction. ${ }^{28-30}$ In patients with significant mitral valve regurgitation, mitral valve repair or replacement might be performed in conjunction with the CSD implantation. These concomitant procedures have been successfully performed in more than 65 patients to date, with slight modifications to the CSD implantation procedure.

Several limitations are inherent in this study, which represented the initial clinical experience for use of the Acorn CorCap CSD in patients with idiopathic or ischemic dilated cardiomyopathy. As such, patients were not randomized, control patients were not enrolled, and blinding was not used. In addition, a complete record of follow-up was not obtainable for some of the patients. Nevertheless, improvements seen in terms of cardiac structure and function were similar to those observed in several animal models of heart failure.

In conclusion, the CSD provides a promising surgical therapy for dilated cardiomyopathy. On the basis of exten- sive preclinical studies, the CSD not only halts ventricular dilation but also appears to promote ventricular reverse remodeling without any safety issues. Similar results are reflected in the initial clinical experience, with up to 2 years of patient follow-up. The CSD appears to be a safe product and might improve cardiac function. On the basis of these studies, randomized clinical trials are currently underway in Europe and North America. This surgical approach provides additional evidence supporting aggressive management of ventricular remodeling in the treatment of heart failure.

\section{References}

1. Baig MK, Mahon N, McKenna WJ, et al. The pathophysiology of advanced heart failure. Heart Lung. 1999;28:87-101.

2. Chien KR. Stress pathways and heart failure. Cell. 1999;98:555-8.

3. Goldstein S, Ali AS, Sabbah H. Ventricular remodeling. Cardiol Clin. 1998;16:623-32.

4. Florea VG, Mareyev VY, Samko AN, et al. Left ventricular remodelling: common process in patients with different primary myocardial disorders. Int J Cardiol. 1999;68:281-7.

5. Lee TH, Hamilton MA, Stevenson LW, et al. Impact of left ventricular cavity size on survival in advanced heart failure. Am J Cardiol. 1993;72:672-6.

6. Vasan RS, Larson MG, Benjamin EJ, et al. Left ventricular dilatation and the risk of congestive heart failure in people without myocardial infarction. N Engl J Med. 1997;336:1350-5.

7. Rector TS, Kubo SH, Cohn JN. Validity of the Minnesota Living with Heart Failure questionnaire as a measure of therapeutic response to enalapril or placebo. Am J Cardiol. 1993;71:1106-7.

8. Ni H, Toy W, Burgess D, et al. Comparative responsiveness of Short-Form 12 and Minnesota Living With Heart Failure Questionnaire in patients with heart failure. J Card Fail. 2000;6:83-91.

9. Havranek EP, Lapuerta P, Simon TA, L'Italien G, Block AJ, Rouleau JL. A health perception score predicts cardiac events in patients with heart failure: results from the IMPRESS trial. J Card Fail. 2001;7: 153-7.

10. Little WC, Downes TR, Applegate RJ. Invasive evaluation of left ventricular diastolic performance. Herz. 1990;15:362-76.

11. Schertel ER. Assessment of left-ventricular function. Thorac Cardiovasc Surg. 1998;46(Suppl 2):248-54.

12. Kern MJ, de Bruyne B, Pijls NH. From research to clinical practice: current role of intracoronary physiologically based decision making in the cardiac catheterization laboratory. J Am Coll Cardiol. 1997;30: 613-20.

13. Verberne HJ, Piek JJ, van Liebergen RA, et al. Functional assessment of coronary artery stenosis by Doppler derived absolute and relative coronary blood flow velocity reserve in comparison with $(99 \mathrm{~m}) \mathrm{Tc}$ MIBI SPECT. Heart. 1999;82:509-14.

14. Konertz W, Shapland JS, Hotz H, et al. Passive containment and reverse remodeling by a novel textile cardiac support device. Circulation. 2001;104(Suppl I):I270-5.

15. Raman JS, Power JM, Buxton BF, et al. Ventricular containment as an adjunctive procedure in ischemic cardiomyopathy: early results. Ann Thorac Surg. 2000;70:1124-6.

16. Raman JS, Hata M, Storer M, et al. The mid-term results of ventricular containment (ACORN WRAP) for end-stage ischemic cardiomyopathy. Ann Thorac Cardiovasc Surg. 2001;7:278-81.

17. Kleber FX, Sonntag S, Rux S, et al. The CorCap \& trade; cardiac support device does not cause pericardial constriction. In press.

18. Chaudhry PA, Mishima T, Sharov VG, et al. Passive epicardial containment prevents ventricular remodeling in heart failure. Ann Thorac Surg. 2000;70:1275-80.

19. Power JM, Raman J, Dornom A, et al. Passive ventricular constraint amends the course of heart failure: a study in an ovine model of dilated cardiomyopathy. Cardiovasc Res. 1999;44:549-55.

20. Sabbah HN, Maltsev VA, Chaudhry PA, et al. Contractile function of 
cardiomyocytes isolated from dogs with heart failure is enhanced after chronic therapy with passive ventricular constraint using the Acorn Cardiac Support Device. Circulation. 1999;100(Suppl):439.

21. Sabbah HN, Gupta RC, Sharov VG, et al. Prevention of progressive left ventricular dilation with the acorn cardiac support device (CSD) downregulates stretch-mediated p21-ras, attenuates myocyte hypertrophy and improves sarcoplasmic reticulum calcium cycling in dogs with heart failure. Circulation. 2000;102(Suppl II):II683.

22. Sabbah HN, Gupta RC, Mishra S, et al. Prevention of progressive left ventricular dilation with the acorn cardiac support device normalizes protein phosphatase activity in dogs with chronic heart failure. Circulation. 2001;104(Suppl II):556.

23. Gupta RC, Sharov VG, Mishra S, et al. Chronic therapy with the acorn cardiac support device (CSD) attenuates cardiomyocyte apoptosis in dogs with heart failure. J Am Coll Cardiol. 2001;37(Suppl A):478A.

24. Saavedra WF, Tunin RS, Paolocci N, et al. Reverse remodeling and enhanced adrenergic reserve from passive external support in experimental dilated heart failure. J Am Coll Cardiol. 2002;39:2069-76.

25. Sabbah H, Chaudhry P, Kleber F, et al. Passive mechanical contain- ment of progressive left ventricular dilation: a surgical approach to the treatment of heart failure. $J$ Heart Failure. 2000;6:115

26. Kleber FX, Sonntag S, Krebs H, et al. Follow-up on passive cardiomyoplasty in congestive heart failure: influence of the Acorn Cardiac Support Device on left ventricular function. J Am Coll Cardiol. 2001; 37(Suppl A):143A.

27. Blondheim DS, Jacobs LE, Kotler MN, et al. Dilated cardiomyopathy with mitral regurgitation: decreased survival despite a low frequency of left ventricular thrombus. Am Heart J. 1991;122:763-71.

28. Kono T, Sabbah HN, Rosman H, et al. Left ventricular shape is the primary determinant of functional mitral regurgitation in heart failure. J Am Coll Cardiol. 1992;20:1594-8.

29. Boltwood CM, Tei C, Wong M, et al. Quantitative echocardiography of the mitral complex in dilated cardiomyopathy: the mechanism of functional mitral regurgitation. Circulation. 1983;68:498-508.

30. Izumi S, Miyatake K, Beppu S, et al. Mechanism of mitral regurgitation in patients with myocardial infarction: a study using real-time two-dimensional Doppler flow imaging and echocardiography. Circulation. 1987;76:777-85

\section{Bound volumes available to subscribers}

Bound volumes of The Journal of Thoracic and Cardiovascular Surgery are available to subscribers (only) for the 2003 issues from the Publisher, at a cost of $\$ 134.00$ for domestic, $\$ 165.85$ for Canadian, and $\$ 155.00$ for international subscribers for Vol 125 (January-June) and Vol 126 (July-December). Shipping charges are included. Each bound volume contains a subject and author index and all advertising is removed. The binding is durable buckram with the Journal name, volume number, and year stamped in gold on the spine. Payment must accompany all orders. Contact Mosby, Subscription Customer Service, 6277 Sea Harbor Dr, Orlando, FL 32887, USA; phone 800-654-2452 or 407-345-4000.

Subscriptions must be in force to qualify. Bound volumes are not available in place of a regular Journal subscription. 